IN MEMORIAM

Fonoaudióloga Edith Schwalm

(1931 - 2017)

Primera directora de la Carrera de Fonoaudiología

Universidad de Chile

Maestra de fonoaudiólogos/as

\title{
Test de articulación a la repetición (TAR): un legado de la profesora fonoaudióloga Edith Schwalm
}

Mariangela Maggiolo L.

Fonoaudióloga Universidad de Chile mmaggiol@med.uchile.cl
"A mi maestra Edith con cariño y reconocimiento por haber podido compartir su vida profesional y haberme hecho parte de esta herencia para la fonoaudiología chilena" 


\section{Antecedentes}

El origen de la fonoaudiología en Chile está ligada a un puñado de nombres, particularmente de mujeres, que forjaron sus cimientos con profunda vocación y profesionalismo. Una de ellas fue la profesora Edith Schwalm Arteaga quien, más allá de haber sido la primera directora de la Escuela de Fonoaudiología de la Universidad de Chile, fue una gran profesional y una mejor maestra.

Destacó entre sus muchas habilidades por darle cabida a su intuición en el tratamiento de los niños y adultos con problemas de lenguaje, virtud que, sumada a su espíritu inquieto, le permitió formular interesantes preguntas que se empeñó en responder con su trabajo asistencial y académico en conjunto con sus pares y estudiantes.

Una de las interrogantes que la inquietaba a comienzos de la década de los ochenta era cómo explicar el fenómeno de lo entonces conocido como "dislalias múltiples". De acuerdo a lo que ella había observado en los niños con problemas de lenguaje, las producciones variadas de los fonemas y de las palabras que ellos realizaban no podían explicarse desde el punto de vista articulatorio solamente, algo más había en esas deformaciones de palabras que trascendía la perspectiva de un análisis puramente fonético. Vale la pena recordar que en esos años era frecuente hacer el diagnóstico de dislalias múltiples en los niños cuya expresión era poco inteligible y evidenciaban "deformaciones de las palabras", especialmente en los que eran diagnosticados con Retraso Simple de Lenguaje (RSL). ¿Cómo podía explicarse, por ejemplo, que un niño dijera “poakatita" en vez de "Caperucita" o "taninedo" por "carabinero"? Obviamente no se trataba sólo de dificultades en los fonemas, sino también en la organización de las palabras: omisiones de sílabas y de fonemas, sustituciones de fonemas, reducción de secuencias consonánticas y vocálicas. Los fenómenos anteriores eran algunos de los registros que se hacían de la expresión fonológica de los niños y que la profesora Schwalm intentaba sistematizar de algún modo. Dicho de otra manera, las deformaciones de palabras asociadas al diagnóstico de dislalias múltiples en los niños con déficit evolutivo del lenguaje podrían considerarse el antepasado de lo que hoy conocemos como dificultades fonológicas o trastorno fonológico. Con certeza en el análisis de la profesora Schwalm ese concepto ya empezaba a construirse.

Uno de los autores de ese tiempo, Charles Bouton, había ya descrito como un síntoma importante de los niños con RSL las deformaciones de palabras a nivel fonológico, lo que provocaba su expresión poco inteligible; problemas en las secuencias fónicas no así en los sonidos aislados, fenómenos como asimilación, reducciones de grupos complejos y metátesis, eran algunas de las descripciones del autor para referirse al desempeño fonológico de estos niños (Bouton, 1976). En ese mismo sentido, otro clásico de la escuela francesa planteaba que se observaban inversiones y simplificaciones fonológicas abundantes y que las palabras no eran más que "una aproximación fonética" (Hécaen y Angelergues, 1965). Parecía, por lo tanto, un desafío del momento aproximarse de modo más específico a estas dificultades y contar con un instrumento que permitiera ponerlas de manifiesto y así contribuir a identificar con más 
precisión a estos niños.

En esos años, la carencia de instrumentos de evaluación del habla (y del lenguaje en general) en nuestro país, motivó a que diversos trabajos de investigación realizados en la Escuela de Fonoaudiología de la Universidad de Chile estuvieran orientados a sistematizar procedimientos evaluativos y a aplicar diversos instrumentos adaptados al medio nacional. Ello tuvo en ese momento un doble propósito: por una parte, permitir una mejor caracterización del habla y del lenguaje infantil en diversos niveles $y$, por otra, posibilitar una mejor identificación de los niños con RSL para efectos de la investigación muy incipiente en aquella época.

En ese contexto, en 1981 bajo la iniciativa de la profesora Schwalm y la profesora María Mercedes Pavez, se realizaron dos estudios que pretendían aportar a una mejor caracterización semiológica de los niños con RSL. Uno de ellos tuvo como objetivo evaluar el desempeño gramatical en niños con RSL a partir de la aplicación del Screening Test of Spanish Grammar (Toronto, 1973) y del Development Assesment Spanish Grammar (DASG) (Toronto, 1976; Barrera, Becerra, Gutiérrez, Marino y Sancho, 1981). El otro estudio se planteó como propósito caracterizar los patrones de error fonológico en niños con RSL (Aguirre, De Barbieri, Gana, Maggiolo y Silva, 1981).

Para llevar a cabo ambas investigaciones y seleccionar adecuadamente la muestra de niños con RSL, fue necesario crear una prueba con el propósito de poner de manifiesto las deformaciones de palabras propias de los niños con este diagnóstico. Lo anterior se efectuó porque no se disponía de instrumentos apropiados ni validados en nuestro medio que permitieran esa caracterización. La idea de estructurar este test fue de la profesora Schwalm. Para ello se basaba en su experiencia de acuerdo a la cual utilizaba una lista de palabras y frases, con la modalidad repetición, para identificar esas deformaciones en los niños que atendía. Sin embargo, en el marco de una investigación y para efectos de seleccionar la muestra en estudio, había que perfeccionar dicha lista. Este antecedente constituye realmente el origen del Test de Articulación a la Repetición (TAR) que paradojalmente, cómo se desprende de lo expuesto, se creó con un propósito diferente al de evaluar la articulación. Es importante destacar que este instrumento es ampliamente usado en nuestro medio en la actualidad.

\section{Características, aplicación interpretación de la prueba}

En este acápite, se incluyen tres aspectos del TAR de acuerdo a su versión original: una descripción del instrumento, cómo se aplica y cómo se interpretan los resultados. Con respecto a cómo se efectúa la evaluación del desempeño obtenido por un niño, conviene precisar que el instrumento se creó con un objetivo funcional en el marco de dos estudios en niños con RSL para identificar sus deformaciones de palabras, por lo que el criterio de interpretación de los resultados refiere a ello de modo muy general y en función de los objetivos de las investigaciones señaladas.

\section{Descripción de la prueba}


La descripción que se incluye a continuación corresponde a la contenida en los trabajos de investigación referidos anteriormente (Aguirre et al., 1981; Barrera et. al, 1981) considerados hasta ahora las fuentes originales donde aparece por primera vez plasmado en su totalidad el instrumento de la profesora Schwalm. La autora de este documento se ha permitido algunos cambios en la redacción y un par de notas que ha estimado pertinente incorporar a modo de explicación.

La Prueba de articulación a la repetición consta de cinco secciones que se presentan a continuación:

\section{Fonemas}

Incluye 124 palabras bisilábicas y trisilábicas que contienen los 17 fonemas consonánticos del sistema fonológico del español según su realización en Chile, ordenados de acuerdo a su zona articulatoria de anterior a posterior. Cada uno de los fonema, se evalúa en lo que se denominaba posición inicial, media, final y trabante y en dos oportunidades por cada posición para mayor confiabilidad del registro del desempeño del niño. Se exceptúan de la evaluación en posición trabante el fonema vibrante múltiple y los fonemas palatales. Es preciso señalar que evaluar los fonemas en todas las posiciones antes mencionadas era un criterio de uso común en las pruebas de evaluación de la época (véase, por ejemplo, Melgar, 1976). Se consideraba posición inicial cuando el fonema iniciaba la primera sílaba de la palabra (ejemplo /d/ en "dama"), medial cuando iniciaba la sílaba ubicada en el centro de un trísilabo (ejemplo /d/ en "cadena") y final cuando iniciaba la silaba final (ejemplo /d/ en "codo"). La posición trabante en cambio correspondía al fonema en posición final de una sílaba, es decir, a la coda silábica (ejemplo /d/ en "admite").

\section{Diptongos (denominados como dífonos vocálicos en el test)}

Incluye 26 palabras que contienen 13 diptongos posibles en español, tanto crecientes (ejemplo "piano") como decrecientes (ejemplo "auto").

\section{Secuencias consonánticas}

Incluye 25 palabras con diferentes secuencias consonánticas homosilábicas en el español tanto en sílaba inicial ("blusa") como final de palabra ("tabla"). Considero oportuno precisar aquí algo que en la versión original no aparece, pero que otorga una explicación al uso sólo de palabras bisílabas en este ítem. Lo anterior se fundamenta en evitar un grado de dificultad mayor a los niños al tener que repetir palabras de mayor metría con sílabas de estructura compleja, puesto que esta doble dificultad podría influir en su desempeño en la emisión de secuencias consonánticas y dificultar el análisis de sus respuestas.

\section{Palabras polisilábicas}

Consta de 10 palabras, 5 de ellas son palabras sólo con sílabas del tipo CV (por ejemplo, "mariposa") y otras 5 que contienen estructuras silábicas que ofrecen mayor dificultad en su realización como secuencias consonánticas homosilábicas (ejemplo "bicicleta") y sílabas con coda silábica (ejemplo "helicóptero"). La elección de la mitad de palabras con estructura silábica más simple y la otra mitad con estructuras más complejas evidencia el equilibrio con el que están seleccionadas 
las palabras estímulo. En este ítem, los polisílabos de estructura silábica simple neutralizan de algún modo el efecto de la complejidad silábica en la producción de palabras más largas por parte de los niños; del mismo modo, los polisílabos que contienen estructuras complejas aportan un grado de dificultad mayor en su producción y evidenciarían una mayor probabilidad de identificar simplificaciones fonológicas en las respuestas de los niños.

\section{Oraciones}

Esta sección incluye 6 oraciones ordenadas de menor a mayor complejidad determinada por la longitud y la estructura gramatical de ellas. A partir de las evidencias actuales en relación a las dificultades que presentan los niños con Trastorno específico del lenguaje con respecto a la repetición de oraciones, el hecho de que en esta prueba se haya incorporado hace más de 35 años un ítem que incluya este tipo de tareas refleja la idea visionaria de la profesora Schwalm en cuanto a lo que ella ya había observado en los niños con problemas de lenguaje. Lo mismo se puede argumentar respecto a la repetición de polisílabos correspondiente a la sección anterior.

\section{Aplicación del TAR}

El instrumento fue diseñado para aplicarse en forma individual considerando las condiciones más adecuadas para ello, es decir evitando distractores auditivos y visuales. El examinador indica al niño/niña poner atención a la palabra que va a decirle porque la debe repetir una vez que la escuche. Se debe tener la precaución de que el evaluado no observe los movimientos articulatorios del examinador. Las repeticiones realizadas por el niño/niña se registran textualmente en la hoja del protocolo en el espacio punteado que se consigna al lado de cada palabra.

Una instrucción similar se proporciona en la sección oraciones, indicando poner atención a las frases, precisando que primero serán cortas y luego frases más largas, las que se deben repetir lo mejor posible una vez que se escuchen. Al igual que en el caso de las palabras, el registro de las repeticiones de las frases se consigna en el espacio punteado que se encuentra a continuación de cada una de ellas.

\section{Interpretación de los resultados}

La evaluación del desempeño obtenido en el TAR es un aspecto que se señala someramente en la versión original del instrumento y alude esencialmente a un criterio cuantitativo y funcional. Dado que no existían antecedentes previos respecto del desempeño de niños con desarrollo típico del lenguaje en esta prueba, era necesario establecer algún criterio cuantitativo para interpretar las respuestas. Se requería también un punto de corte como referencia a la normalidad, en particular si se considera que los niños con RSL incluidos originalmente en el estudio de Aguirre et al. (1981) tenían 4 años, por lo que era factible que aquellos con desarrollo típico de la misma edad también presentaran deformaciones de palabras (hoy el concepto de deformación correspondería a lo que se conoce como procesos de simplificación fonológica).

Se determinó asignar un punto por la presencia de deformaciones de palabras en las respuestas a cada ítem que evalúa los fonemas, los diptongos, las secuencias consonánticas y los polisílabos. No se 
consideraron los ítems de la sección repetición de oraciones. La versión original del test no señala explícitamente cómo se realizó el conteo de deformaciones, es decir si una palabra deformada equivalía a un punto, sin embargo ese fue el criterio utilizado $^{1}$. Es importante aclarar este aspecto, dado que desde la perspectiva de los procesos fonológicos de simplificación puede coexistir más de un proceso en una misma palabra y cada uno de ellos se identifica como tal (hecho que no se consideraba en el TAR en el cual solo se consignaba el número de palabras deformadas).

Una vez establecido el criterio de interpretación de las respuestas se realizó un pilotaje con 10 niños con desarrollo típico entre 4 y 4.11 años estableciéndose a partir de los resultados obtenidos que 20 deformaciones de palabras sería el puntaje máximo para estimar que un niño tenía un desempeño normal. De este modo, los niños que obtuvieran un puntaje mayor a 20 en el TAR serían considerados con dificultades de lenguaje.

\section{La versión reducida del TAR}

En el año 1987 se planteó una versión reducida de la prueba (Barrios, Benavides, Benavides, Dellafiori, 1987) puesto que su aplicación resultaba excesivamente larga, particularmente para los niños pequeños. Lo anterior tiene sentido desde un punto de vista de la práctica profesional ya que contar con un instrumento de fácil y rápida aplicación es más útil en la evaluación de los niños en contextos de intervención.

Se redujo el número de ítems que evalúan

\footnotetext{
${ }^{1}$ La autora de este artículo fue co-autora del trabajo de Aguirre y por ende puede consignar el criterio utilizado.
}

fonemas, diptongos, secuencias consonánticas y polisílabos. En lo que respecta a las oraciones se mantuvieron las seis que conforman la sección original, sólo que en la sexta se agregó el sintagma "su mamá" (“Juanito se metió debajo de la cama para que no lo pillara su mamá") para diferenciarla en cuanto a longitud de la oración número cinco (“El mono que estaba dentro de la jaula se comió mi maní"). La reducción del TAR no cambió su organización global en cuanto al orden de los ítems. Para información de la prueba original y reducida véanse los anexos 1 y 2 respectivamente.

Así, los ítems de la nueva versión del TAR quedaron como sigue: 63 palabras bisilábicas para la evaluación de los fonemas; 6 palabras para evaluar los diptongos (que en esta versión se denominaron dífonos vocálicos), 13 palabras para medir los dífonos consonánticos (correspondientes a secuencias consonánticas homosilábicas) y 6 palabras para los polisílabos.

Como se ha podido inferir de lo señalado con respecto a la versión original, una de las debilidades del TAR es no contar con puntajes normativos provenientes de muestras significativas de población típica. El mismo problema se evidencia en su versión reducida que aunque cuenta con un puntaje de referencia éste también se obtuvo a partir de un pilotaje con un número reducido de niños con desarrollo típico. Para esta versión, los puntajes se obtuvieron a partir de un piloto efectuado con 22 niños con desarrollo típico entre 4 y 4.11 años de nivel socioeconómico bajo. Es importante señalar aquí que este grupo de niños fue seleccionado de acuerdo a las siguientes características: coeficiente intelectual dentro de rangos normales; audición 
compatible con la adquisición del lenguaje; indemnidad neurológica; indemnidad anátomofuncional de los órganos fonoarticulatorios y tener como lengua materna el español. La evaluación del C.I. y la evaluación neurológica fueron realizadas por un profesional psicólogo y neurólogo respectivamente.

A partir del desempeño de los niños de este grupo se obtuvieron los siguientes puntajes de referencia para la versión reducida:

- $\quad$ Puntaje promedio: 82.86 puntos

- $\quad$ Desviación estándar: 3.5

- $\quad$ Rango de normalidad: entre 79.36 y 86.36 puntos

Aun cuando el trabajo de Barrios et al. (1987) no lo menciona, se infiere que los puntajes señalados se obtuvieron sólo de contar las respuestas de los niños en los ítems correspondientes a los fonemas, dífonos vocálicos, consonánticos y polisílabos. Lo anterior dado que el total de palabras considerando sólo estos ítems es de 88. Si cada repetición de palabra es correcta, el puntaje máximo que puede obtener un niño es 88 puntos. No hay evidencia que las frases se hayan considerado para la obtención de estos puntajes al igual que en la primera versión.

\section{EI TAR bajo la perspectiva de las nuevas tendencias para evaluar la producción fonético-fonológica}

De lo señalado con respecto al "Test de articulación a la repetición" y considerando la tendencia actual es posible inferir que es un instrumento con un potencial importante en cuanto a la información que puede proporcionar en relación al desempeño fonético-fonológico infantil. Desde el propósito de este artículo, lo más relevante aún es destacar que la profesora Schwalm lo vislumbró de alguna manera hace más de treinta años.

Nadie discute que los niños con problemas de lenguaje presentan dificultades en la producción fonético- fonológica y parece arbitrario analizar esas dificultades desde visiones teóricas separadas, hay suficiente evidencia científica al respecto.

Históricamente los límites entre la fonética y la fonología han sido objeto de discusión; en algunas épocas se les ha considerado más próximos, en otras más lejanos. Lo que no es discutible es la influencia que han tenido en la formación de los fonoaudiólogos y por ende en lo que respecta a los fundamentos teóricos de la evaluación e intervención de los niños con dificultades fonéticofonológicas (Susanibar et al., 2016).

En la actualidad, la integración de perspectivas, metodologías de investigación, formas de abordaje del objeto de estudio demuestra que parece ser el punto de vista más razonable para el análisis de la producción fonético-fonológica. Lo anterior, no solo permite explicar más apropiadamente lo que ocurre en los niños con desarrollo típico, sino que también posibilita un mejor abordaje de las dificultades en este ámbito que pueden presentarse en los trastornos evolutivos del lenguaje y del habla.

En ese mismo sentido, la ASHA (2017) desde hace más de una década ha propuesto una entidad denominada "Trastorno de los sonidos del habla" (TSH). Bajo este concepto se incluyen todas las combinaciones de déficit de la percepción, de la producción motora $\mathrm{y} / \mathrm{o}$ de la representación fonológica de los sonidos y segmentos del habla; así 
como de las reglas fonotácticas que rigen la estructura silábica, la acentuación y la prosodia que puedan impactar en la inteligibilidad del habla.

Aun cuando el TSH se relaciona frecuentemente con trastornos articulatorios de etiologías conocidas, con frecuencia no es posible distinguir si en la dificultad que presenta un niño subyace más bien una causa articulatoria o fonológica (por ende, ligada más bien al lenguaje). Con respecto de esto último, se sabe que las dificultades fonológicas se relacionan con problemas en la representación mental de la palabra, por lo que dentro de este amplio concepto cabrían ambos tipos de dificultades.

Más allá de la discusión y eventuales discrepancias que lo señalado podría generar, lo esencial de este manuscrito es revisitar un instrumento creado hace más de tres décadas en nuestro país. Cuando la profesora Schwalm pensó en darle estructura a la prueba tenía un propósito muy preciso y acotado: contribuir a la selección de un grupo de niños con RSL que constituirían una muestra en dos estudios que se estaban realizando en paralelo en la Escuela de Fonoaudiología. Con posterioridad el TAR se ha seguido utilizando en el ámbito nacional, como una forma de evaluar el desempeño articulatorio de los niños y en menor grado para identificar dificultades fonológicas, particularmente antes de la aparición de instrumentos específicos para evaluar procesos fonológicos de simplificación con normas chilenas. Su interpretación, quién sabe si por razones históricas, contingentes, incluso de recursos, ha permanecido igual, es decir, sin contar con normas de referencia nacional estadísticamente adecuadas. Así, los resultados del desempeño de los niños evaluados se constituyen más bien en una pauta orientadora que se contrasta con lo que cada profesional espera de acuerdo a su conocimiento del desarrollo típico de los niños.

Personalmente creo que una manera de honrar la memoria de una de las maestras de la fonoaudiología chilena, Sra. Edith Schwalm, sería iniciar el camino de revisar el TAR con la mirada actual acerca de los déficits fonético-fonológicos. Un proyecto con este propósito debería considerar grupos de niños con diversas características sociodemográficas (edad, nivel sociocultural), definir con precisión criterios de interpretación de las respuestas, realizar el análisis estadístico que se requiere, incorporar un manual de aplicación, entre otros aspectos que involucran la creación de un test estandarizado.

Con ello se podría contar con una versión formal del instrumento, constituyéndose en un aporte útil y trascendente para nuestro ejercicio profesional. 


\section{Referencias}

American Speech-Language-Hearing Association (ASHA). (2017). Speech Sound Disorders: Articulation and Phonological Processes. Revisado desde: https://www.asha.org/public/speech/disorders/SpeechSou ndDisorders/

Aguirre, P., De Barbieri, Z., Gana, C., Maggiolo, M. y Silva, C. (1981). Patrones de error fonológicos en niños con Retraso Simple de Lenguaje. Seminario para optar al título de Fonoaudiólogo, Facultad de Medicina, Universidad de Chile.

Barrera, J., Becerra, N., Gutiérrez, M., Marino, E. y Sancho, M. (1981). Evaluación del desempeño gramatical en niños con Retraso Simple de Lenguaje. Seminario para optar al título de Fonoaudiólogo, Facultad de Medicina, Universidad de Chile.

Barrios, P., Benavides, C., Benavides, L. y Dellafiori. C. (1987). Evaluación de la conducta lingüística de niños de centros abiertos de 4 a 4.11 años. Seminario para optar al título de Fonoaudiólogo, Facultad de Medicina, Universidad de Chile.

Bouton, C. (1976). Desarrollo del lenguaje, aspectos normales y patológicos. Ed. Huemul, Buenos Aires.

Hécaen, H. y Angelergues, R. (1965). Pathologie du Langage. Libraire Larousse, 1 a edición, París.

Melgar, M. (1976). Cómo detectar al niño con problemas de habla. Editorial Trillas, 1a edición, México.

Susanibar, F., Dioses, A., Marchesan, I., Guzmán, M., Leal, G., Guitar, B. y Junqueira, A. (2016). Trastornos del Habla, de los fundamentos a la evaluación. Editorial EOS, Madrid.

Toronto, A. (1973). Screening Test of Spanish Grammar. Northwestern University Press, Evanston III.

Toronto, A. (1976). Developmental Assessment of Spanish Grammar. Journal of Speech and Hearing Disorders, Vol. 41, № 2, pp 150-171. 
Anexo 1 (imagen escaneada desde documento original)

NOMBRE :
EDAD :
FECHA :

EXAMINADOR :

\section{EXAMEN DE ARTICULACION}

\begin{tabular}{|c|c|c|c|c|}
\hline POSICION & inicial & medial & final & trabando \\
\hline FONEMAS & & & & \\
\hline Bilabiale & & & & \\
\hline$/ \mathrm{b} /$ & $\begin{array}{l}\text { bote..... } \\
\text { bala..... }\end{array}$ & $\begin{array}{l}\text { cabeza.... } \\
\text { tabaco.... }\end{array}$ & $\begin{array}{l}\text { nube..... } \\
\text { tubo..... }\end{array}$ & $\begin{array}{l}\text { objeto....... } \\
\text { submarino.... }\end{array}$ \\
\hline$/ p /$ & $\begin{array}{l}\text { pato..... } \\
\text { pesa.... }\end{array}$ & $\begin{array}{l}\text { zapato..... } \\
\text { tapado..... }\end{array}$ & $\begin{array}{l}\text { copa..... } \\
\text { sopa.... }\end{array}$ & $\begin{array}{l}\text { apto } \ldots . . . . \\
\text { séptimo..... }\end{array}$ \\
\hline$/ \mathrm{m} /$ & $\begin{array}{l}\operatorname{mano} . . . . \\
\text { mesa..... }\end{array}$ & $\begin{array}{l}\text { camisa..... } \\
\text { camote..... }\end{array}$ & $\begin{array}{l}\text { suma.... } \\
\text { I omo.... }\end{array}$ & $\begin{array}{l}\text { campo....... } \\
\text { temprano..... }\end{array}$ \\
\hline
\end{tabular}

Labio-dental :

/f/ foca.... búfalo.... café.... aftosa......

fino.... zafiro.... mofa.... difteria....

Dentales :

/d/

dama..... cadena..... codo.... pared.......

dato..... madera.... nudo.... admite......

$/ \mathrm{t} /$

tapa.... botella... mata.... etna .......

tina.... tetera.... lote........

Alveolares :

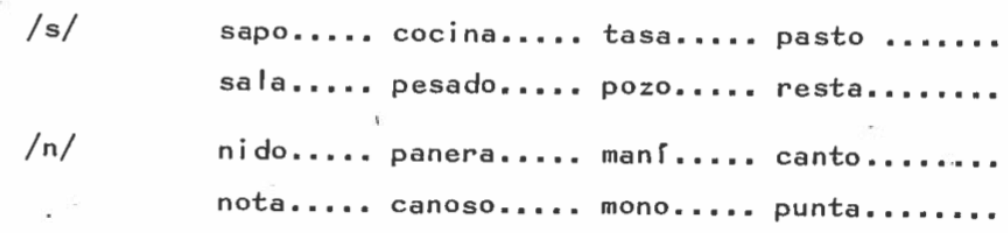




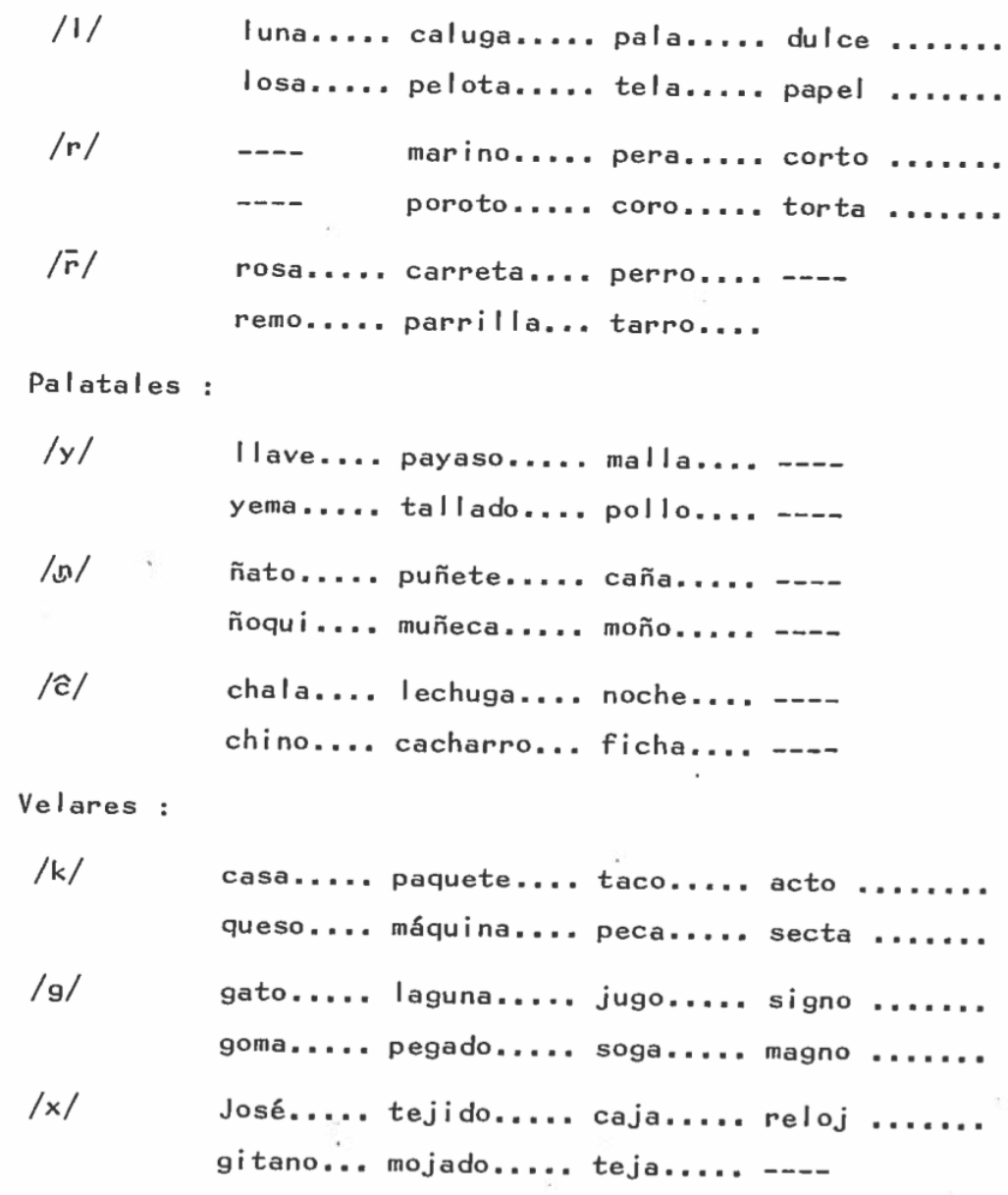

DIFONOS VOCALICOS :
piano.... pie....... ciudad... suave ......
diario... tiene..... diuca.... guata ......
violin... nuevo..... fui .... cuota ......
piojo... fuego..... ruin.... fatur ......
vaina.... peineta.... boina... auto .......
laico... rey ...... moisés... pauta....... 


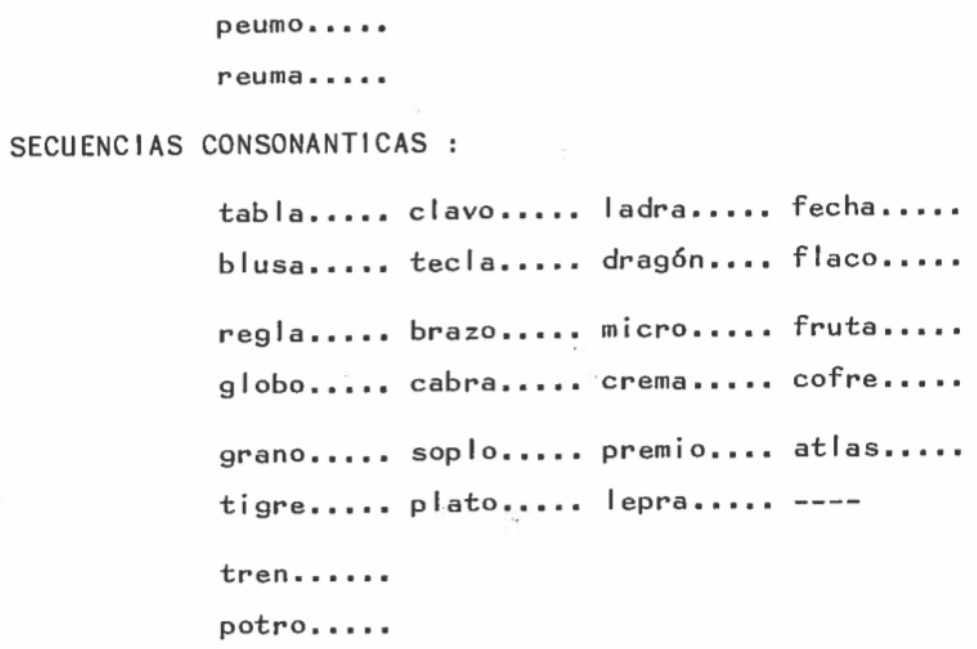

POLISILABICAS :

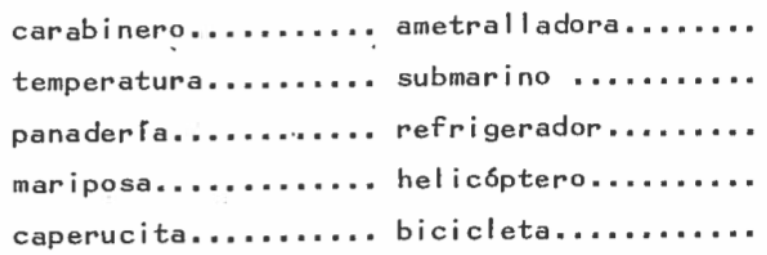

ORACIONES :

1.- El perro salta $\ldots \ldots \ldots \ldots \ldots \ldots \ldots \ldots$.

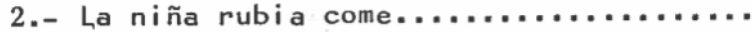

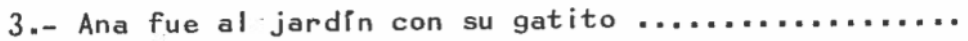

4.- La guagua Iloraba porque tenía hambre............

5.- El mono que estaba dentro de la jaula se comi $6 \mathrm{mi}$

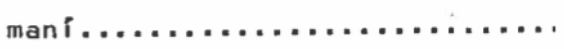

6.- Juanito se meti6 debajo de la cama para que no lo

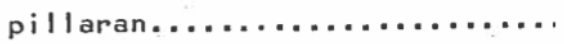

Número total de deformaciones ........... 
Anexo 2 (imagen escaneada desde documento original)

NOMBRE :

FECHA :

FONEMAS

Bilabiales:

/b/

$/ p /$

/m/

Labio-dental:

/f/

Dentales:

/d/

$/ t /$

Alveolares:

/s/

/n/

$11 /$

$/ r /$

$/ r /$

Palatales:

$/ y /$

/ng/

/il
EDAD :

EXAMINADOR:

EXAMEN DE ARTICULACION

inicial medial final trabando bote.... cabeza... nube.... objeto...

pato..... zapato... copa..... apto.....

mano..... camisa... suma.... campo....

foca..... búfalo... café..... aftosa...

dama..... cadena... codo..... pared....

tapa..... botella... mata..... etna.....

sapo.... cocina... taza.... pasto....

nido..... panera... mani.... canto....

luna..... caluga... pala.... dulce....

poroto... coro..... torta....

rosa..... carreta... perro.... -.-

Ilave.... payaso... malla.... ...

ก̃ato..... puñete... cúna........

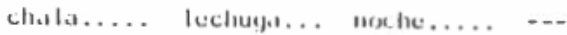


Velares:

$/ \mathrm{k} /$

$/ g /$

$/ \mathrm{j} /$

DIFONOS VOCALICOS:

DIFONOS CONSONANTICOS:

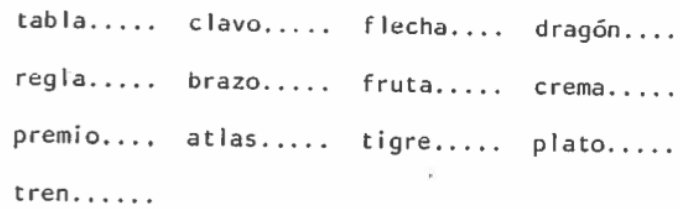

POLISILABICAS:

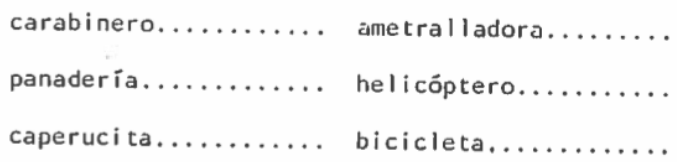

FRASES:

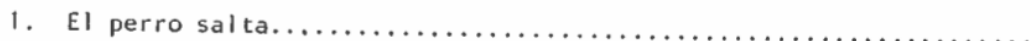

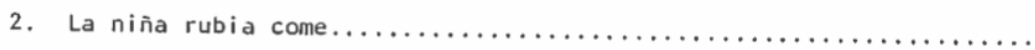

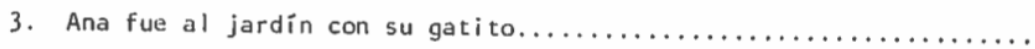

4. La guagua lloraba porque tenía hambre....................

5. El mono que estaba dentro de la jaula se comió mi maní.............

6. Juani to se metió debajo de la cama para que no lo pillara su mamá.. 